\title{
EFFECT OF HEAT TRANSFER ON THERMAL STRESSES IN AN ANNULAR HYPERBOLIC FIN: AN APPROXIMATE ANALYTICAL SOLUTION
}

\author{
Ashis Mallick, Rajiv Ranjan, Prabir Kumar Sarkar \\ Department of Mechanical Engineering, Indian School of Mines, Dhanbad, India \\ e-mail: mal123_us@yahoo.com
}

An approximate analytical solution is presented for thermal stresses in an annular convectiveconductive fin of a hyperbolic profile with temperature dependent thermal conductivity. The classical thermo-elasticity theory coupled with the ADM based polynomial form of temperature field is employed for an approximate analytical solution of thermal stresses. The influence of thermal parameters, i.e. variable thermal conductivity, the thermo-geometric parameter and the non-dimensional coefficient of thermal expansion on temperature and sttress fields are investigated. The results for the stress field obtained from the ADM based solution are compared with those available in literature and found to be in close agreement.

Keywords: thermal stresses, Adomian decomposition method (ADM), variable thermal conductivity

\section{Nomenclature}

\begin{tabular}{|c|c|}
\hline$r_{a}, r_{b}$ & - inner and outer radius, respectively \\
\hline$t$ & - thickness of the fin \\
\hline$h_{a}$ & - fin thickness at base \\
\hline$h$ & - convective heat transfer coefficient \\
\hline$C_{1}$ & - profile function, $h_{a} r_{a}$ \\
\hline$k(T)$ & - variable thermal conductivity \\
\hline$k_{a}$ & - thermal conductivity at ambient temperature \\
\hline$\kappa$ & - parameter describing variation of thermal conductivity \\
\hline$\beta$ & $\begin{array}{l}\text { dimensionless parameter describing variation of thermal conductivity, } \\
\kappa\left(T_{a}-T_{\infty}\right)\end{array}$ \\
\hline$T, T_{a}, T_{\infty}$ & - fin, base and ambient temperature, respectively \\
\hline$r, \phi$ & - polar coordinates \\
\hline$\alpha$ & - linear coefficient of thermal expansion \\
\hline$E$ & - Young's modulus \\
\hline$\sigma_{r}, \sigma_{\phi}, \varepsilon_{r}, \varepsilon_{\phi}$ & - radial and tangential stress and strains, respectively \\
\hline$C, D_{1}, D_{2}, \eta, A$ & A, $B-$ constants \\
\hline$\psi$ & - thermo-geometric parameter, $\sqrt{2 h r_{a}^{2} /\left(k_{\infty} h_{a}\right.}$ \\
\hline$\xi, \xi_{1}$ & - dimensionless radius of fin, $\xi=\left(r-r_{a}\right) / r_{a}, \xi_{1}=\xi+1$ \\
\hline$R$ & - dimensionless outer radius, $R=r_{b} / r_{a}$ \\
\hline$\theta$ & - dimensionless temperature, $\theta=\left(T-T_{\infty}\right) /\left(T_{a}-T_{\infty}\right)$ \\
\hline $\bar{\sigma}_{r}, \bar{\sigma}_{\phi}$ & - dimensionless radial and tangential stress, $\sigma_{r} / E$ and $\sigma_{\phi} / E$ \\
\hline$\chi$ & - dimensionless coefficient of thermal expansion, $\alpha\left(T_{a}-T_{\infty}\right)$ \\
\hline$\nu$ & - Poisson's ratio \\
\hline$A_{c}, d A_{s}$ & - cross sectional area of fin amd elemental surface area of fin, respectively \\
\hline & - profile parameter \\
\hline$A_{p}, B_{p}$ & - Adomian polynomials \\
\hline
\end{tabular}




\section{Introduction}

The rapid heat dissipation from a surface to the surroundings is required in many engineering and industrial applications such as heat exchangers, semiconductors, transformers, motors and many other electrical, electronical and mechanical components. A fin is an extended surface frequently used for direct heat dissipation from a hot surface to its surroundings (Kern and Kraus, 1972; Kraus et al., 2001).

Thermal analyses of the fin with various geometries are presented by many researchers (Yeh, 1997; Mokheimer, 2002). However, most of the available works focus on straight fins for their ease in manufacturing and mathematical formulation. Several mathematical techniques like Adomian's Decomposition Method (ADM), Homotopy Perturbation Method (HPM), Variational Iteration Method (VIM), and Deferential Transformation Method (DTM) can be found in use by many researchers to obtain semi-analytical solutions to nonlinear heat equations for fins (Miansari et al., 2008; Moradi, 2011). The improvement in heat transfer is shown mainly to be associated with their surface modification. So, an enhancement in the heat transfer rate critically depends on the selection of correct fin shape. Radial shape is observed to outperform the straight fin in respect of heat transfer ability (Behnia et al., 1998). A hyperbolic profile contains even larger surface area to yield more heat dissipation from the surface to the surroundings. Thus, a radial fin with a hyperbolic profile would be the most preferred shape for a better heat transfer rate with less material involved.

Recently, the heat transfer in an annular fin with a hyperbolic profile was presented by Aksoy (2013). Non-uniform temperature distributions during the heat transfer process induce thermal stresses in the fin material. Thermal stresses are responsible for various mechanical failures, i.e., crack propagation, creep and fatigue failure that can reduce the fin life. The study of thermal stresses in them is, therefore, important to prevent their early damage. So far, only few authors (Chiu and Chen, 2002; Mallick et al., 2015) have reported the analysis of stresses developed due to variation of the temperature gradient in an annular fin, and these papers mainly focus on uniform thickness of the fins. A careful review of the published literature reveal that till date no work reports theoretical studies for thermal stresses in an annular radial fin of a hyperbolic profile with the consideration of variable thermal conductivity.

In this paper, a novel analytical approach is used to predict a near closed form solution for thermal stresses in an isotropic homogeneous annular fin with a hyperbolic profile. A variable thermal conductivity parameter is considered in the analysis. The temperature field is obtained by solving a non-linear steady sate heat conduction-convection equation of a fin using the Adominan Decomposition Method. The solution for the temperature field is expressed in a polynomial form. The plane stress condition in a rotational symmetric geometry with respect to its axis is considered in this study. A classical thermo-elasticity relation coupled with the solution of the temperature field is employed to obtain the stress field. The study includes the affect of various non-dimensional parameters such as thermal conductivity parameter $(\beta)$, the thermo-geometric fin parameter $(\psi)$, the coefficient of thermal expansion $(\chi)$ and Poisson's ratio $(\nu)$ on the stress field. The results are compared with those given by Chiu and Chen (2002) to resolve the accuracy of the present method. The analyses presented envisage an improved fin design process.

\section{Problem description and governing equations of heat transfer}

An axisymmetric undeformed annular fin of a hyperbolic profile (Fig. 1) with variable thermal conductivity $(\mathrm{k}(\mathrm{T}))$ is considered. The fin is exposed to a conductive-convective environment at a constant ambient temperature and the tip of the fin is maintained to be well-insulated 
with a traction free condition. As thickness of the fin is relatively small compared to its radial dimension, the temperature distribution and the stress field can be assumed to vary in the radial direction only.

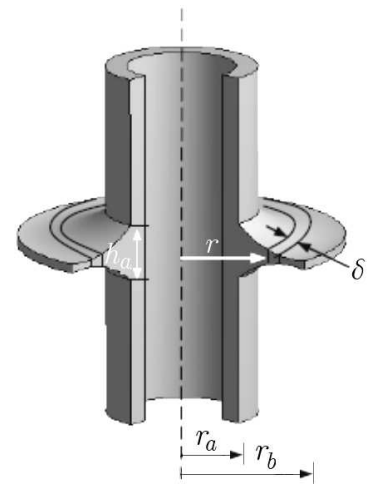

Fig. 1. Geometry of an annular fin with a hyperbolic profile

The profile function for fin tapering from the base to tip is given by

$$
t=h_{a}\left(\frac{r}{r_{a}}\right)^{n} \quad \text { with } \quad n \geqslant 1
$$

where $n$ is the profile parameter.

The steady state energy balance equation for the axisymmetric annular fin is expressed (Mokheimer, 2002) as

$$
\frac{d}{d r}\left(k A_{c} \frac{d T}{d r}\right)-\frac{h d A_{s}}{d r}\left(T-T_{\infty}\right)=0
$$

where $k=k_{a}\left[1+\kappa\left(T-T_{\infty}\right)\right]$. The notations used in Eq. (2.1) and Eq. (2.2) are defined in the nomenclature.

The fin profile becomes hyperbolic when the profile parameter $n=-1$. Equation (2.2) can be expressed in the following dimensionless form

$$
\frac{d^{2} \theta}{d \xi^{2}}+\beta \theta \frac{d^{2} \theta}{d \xi^{2}}+\beta\left(\frac{d \theta}{d \xi}\right)^{2}-\psi^{2}(1+\xi) \theta=0 \quad \text { with } \quad 0 \leqslant \xi \leqslant R-1
$$

where

$$
\theta=\frac{T-T_{\infty}}{T_{a}-T_{\infty}} \quad \beta=\kappa\left(T_{a}-T_{\infty}\right) \quad \xi=\frac{r-r_{a}}{r_{a}} \quad R=\frac{r_{b}}{r_{a}} \quad \psi=\sqrt{\frac{2 h r_{a}^{2}}{k_{a} h_{a}}}
$$

are non-dimensional parameters.

In order to evaluate the temperature distribution, the following non-dimensional boundary conditions are employed

$$
\xi= \begin{cases}0 & \text { that } \quad \theta=1 \\ R-1 & \text { that } \frac{d \theta}{d \xi}=0\end{cases}
$$

\section{Adomian Decomposition Method to solve the heat transfer equation}

Adomian Decomposition Method (ADM) is adopted to evaluate the non-dimensional temperature field. In ADM, the nonlinear ordinary and partial differential equations are represented in an operator form (Adomian, 1988)

$$
L u+R u+N u=g
$$


where $L$ is an $n$-th order invertible linear differential operator $\left(L=d^{n} / d \xi^{n}\right), R$ is a linear differential operator of an order less than $L$, and $N$ represents a nonlinear operator that enables $N u$ to be decomposed into an infinite series of Adomian polynomials.

Applying ADM, the governing equation for the temperature field (Eq. 2.3) can now be expressed as

$$
L \theta=\psi^{2} \theta+\psi^{2} \xi \theta-\beta(N A)-\beta(N B)
$$

where

$$
N A=\theta \theta^{\prime \prime}=\sum_{p=0}^{\infty} A_{p} \quad N B=\left(\theta^{\prime}\right)^{2}=\sum_{p=0}^{\infty} B_{p}
$$

are the nonlinear terms. The Adomian polynomials $A_{n}$ and $B_{n}$ are estimated as follows

$$
\begin{aligned}
& \left\{\begin{array}{l}
A_{0} \\
A_{1} \\
A_{2} \\
A_{3} \\
A_{4} \\
\cdot \\
\cdot
\end{array}\right\}=\left[\begin{array}{ccccccc}
\theta_{0} & & & & & \\
\theta_{1} & \theta_{0} & & & & \\
\theta_{2} & \theta_{1} & \theta_{0} & & & \\
\theta_{3} & \theta_{2} & \theta_{1} & \theta_{0} & & \\
\theta_{4} & \theta_{3} & \theta_{2} & \theta_{1} & \theta_{0} & \\
\cdot & \cdot & \cdot & \cdot & \cdot & \cdot \\
\cdot & \cdot & \cdot & \cdot & \cdot & \cdot
\end{array}\right]\left\{\begin{array}{c}
\theta_{0}^{\prime \prime} \\
\theta_{1}^{\prime \prime} \\
\theta_{2}^{\prime \prime} \\
\theta_{3}^{\prime \prime} \\
\theta_{4}^{\prime \prime} \\
\cdot \\
\cdot
\end{array}\right\} \\
& \left\{\begin{array}{c}
B_{0} \\
B_{1} \\
B_{2} \\
B_{3} \\
B_{4} \\
\cdot \\
\cdot
\end{array}\right\}=\left[\begin{array}{ccccccc}
\theta_{0}^{\prime} & & & & & \\
\theta_{1}^{\prime} & \theta_{0}^{\prime} & & & & \\
\theta_{2}^{\prime} & \theta_{1}^{\prime} & \theta_{0}^{\prime} & & & \\
\theta_{3}^{\prime} & \theta_{2}^{\prime} & \theta_{1}^{\prime} & \theta_{0}^{\prime} & & \\
\theta_{4}^{\prime} & \theta_{3}^{\prime} & \theta_{2}^{\prime} & \theta_{1}^{\prime} & \theta_{0}^{\prime} & \\
\cdot & \cdot & \cdot & \cdot & \cdot & \cdot \\
\cdot & \cdot & \cdot & \cdot & \cdot & \cdot
\end{array}\right]\left\{\begin{array}{l}
\theta_{0}^{\prime} \\
\theta_{1}^{\prime} \\
\theta_{2}^{\prime} \\
\theta_{3}^{\prime} \\
\theta_{4}^{\prime} \\
\cdot \\
\cdot
\end{array}\right\}
\end{aligned}
$$

where $(\cdot)^{\prime}$ and $(\cdot)^{\prime \prime}$ are $d / d \xi$ and $d^{2} / d \xi^{2}$, respectively. An inverse operator $L^{-1}$ can conveniently be used as a two-fold identifying integral in both sides of Eq. (3.2). Applying the Maclaurin series, yields

$$
\theta=\theta(0)+\xi \frac{d \theta(0)}{d \xi}+\psi^{2}\left(L^{-1} \sum_{p=0}^{\infty} \theta_{p}\right)+\psi^{2}\left(L^{-1} \sum_{p=0}^{\infty} \xi \theta_{p}\right)-\beta\left(L^{-1} \sum_{p=0}^{\infty} A_{p}\right)-\beta\left(L^{-1} \sum_{p=0}^{\infty} B_{p}\right)
$$

The first two terms of the right-hand side of Eq. (3.4) can be defined by

$$
\theta_{0}=1+C \xi
$$

where $C$ is the integral constant.

Considering a finite series of the order $p$, the higher order terms in Eq. (3.4) are obtained recursively as

$$
\theta_{p+1}=\psi^{2}\left(L^{-1} \sum_{p=0}^{\infty} \theta_{p}\right)+\psi^{2}\left(L^{-1} \sum_{0}^{p} \xi \theta_{p}\right)-\beta\left(L^{-1} \sum_{0}^{p} A_{p}\right)-\beta\left(L^{-1} \sum_{0}^{p} B_{p}\right)
$$

with $p \geqslant 0$. 
In the present analysis, the estimation of the first four significant terms, i.e, $n=0$ to 3 , of the temperature field is now expressed in the following form

$$
\begin{aligned}
& \theta_{1}=-\beta L^{-1} A_{0}-\beta L^{-1} B_{0}+\psi^{2} L^{-1}\left[(1+\xi) \theta_{0}\right] \\
& \theta_{2}=-\beta L^{-1} A_{1}-\beta L^{-1} B_{1}+\psi^{2} L^{-1}\left[(1+\xi) \theta_{1}\right] \\
& \theta_{3}=-\beta L^{-1} A_{2}-\beta L^{-1} B_{2}+\psi^{2} L^{-1}\left[(1+\xi) \theta_{2}\right] \\
& \theta_{4}=-\beta L^{-1} A_{3}-\beta L^{-1} B_{3}+\psi^{2} L^{-1}\left[(1+\xi) \theta_{3}\right]
\end{aligned}
$$

The total temperature field can now be estimated from Eqs. (3.5) and (3.7) to yield a polynomial form

$$
\theta=\sum_{i=0}^{m} K_{i} \xi^{i} \quad \text { with } \quad 0 \leqslant \xi \leqslant R-1
$$

where $K_{i}$ are constants. The estimation of $K_{i}$ are shown in Appendix.

\section{Thermal stress formulation}

Using the plane stress assumption $\left(\sigma_{z} \cong 0\right)$, the stress-displacement relations in the axisymmetric case (Timoshenko and Goodier, 1970) are

$$
\begin{aligned}
& \sigma_{r}=\frac{E}{1-\nu^{2}}\left[\left(\frac{d u_{r}}{d r}-\alpha T\right)+\nu\left(\frac{u_{r}}{r}-\alpha T\right)\right] \\
& \sigma_{\phi}=\frac{E}{1-\nu^{2}}\left[\left(\frac{u_{r}}{r}-\alpha T\right)+\nu\left(\frac{d u_{r}}{d r}-\alpha T\right)\right]
\end{aligned}
$$

Following classical theory of elasticity, the equation of equilibrium in the polar coordinate system for a variable thickness profile is given as

$$
\frac{d \sigma_{r}}{d r}+\frac{\sigma_{r}}{t} \frac{d t}{d r}+\frac{\sigma_{r}-\sigma_{\phi}}{t r}=0
$$

Equations (4.1) and (4.2) yield the equation of equilibrium in terms of the displacement field

$$
\frac{d^{2} u}{d r^{2}}+\frac{1+n}{r} \frac{d u}{d r}+\frac{(\nu n-1) u}{r^{2}}-(1+\nu) \alpha\left(\frac{d T}{d r}+\frac{T}{r} n\right)=0
$$

Introducing a new non-dimensional radius, $\xi_{1}=r / r_{a}$, the following termo-elastic equation of equilibrium is obtained

$$
\frac{d^{2} u}{d \xi_{1}^{2}}+\frac{1+n}{\xi_{1}} \frac{d u}{d \xi_{1}}+\frac{(\nu n-1) u}{\xi_{1}^{2}}-(1+\nu) r_{a} \alpha\left[\left(T_{a}-T_{\infty}\right) \frac{d \theta}{d \xi_{1}}+\left(T_{a}-T_{\infty}\right) n \frac{\theta}{\xi_{1}}\right]=0
$$

The temperature field in Eq. (3.8) is now modified by the new non-dimensional radius $\xi_{1}$ and can be expressed in an analogous form

$$
\theta=\sum_{i=0}^{m} L_{i} \xi_{1}^{i} \quad 1 \leqslant \xi_{1} \leqslant R
$$

Equations (4.4) and (4.5) give together a new form of the equilibrium equation

$$
\frac{d^{2} u}{d \xi_{1}^{2}}+\frac{1+n}{\xi_{1}} \frac{d u}{d \xi_{1}}+\frac{(\nu n-1) u}{\xi_{1}^{2}}=(1+\nu) r_{a} \chi\left[\sum_{i=0}^{m}(i+n) L_{i} \xi_{1}^{i-1}\right]
$$

where $\chi=\alpha\left(T_{b}-T_{\infty}\right)$ is a non-dimensional coefficient of thermal expansion. 
The complete solution to Eq. (4.6) consists of a homogeneous and a particular solution, and is given as

$$
u=D_{1} \xi_{1}^{\eta_{1}}+D_{2} \xi_{1}^{\eta_{2}}+\sum_{i=0}^{m}(1+\nu) \chi r_{a} \frac{L_{i}(i+n)}{n(1+i+\nu)+i(i+2)} \xi_{1}^{i+1}
$$

where $D_{1}, D_{2}$, and $\eta_{1,2}=-(n / 2) \pm \sqrt{1-\nu n+n^{2} / 4}$ are constants. The stress field Eq. (4.1) can now be expressed in the non-dimensional form

$$
\begin{aligned}
& \bar{\sigma}_{r}=\left[\left(\frac{d u}{d \xi_{1}}+\nu \frac{u}{\xi_{1}}\right) \frac{1}{r_{a}}-\chi(1+\nu) \theta\right] \frac{1}{1-\nu^{2}} \\
& \bar{\sigma}_{\phi}=\left[\left(\frac{u}{\xi_{1}}+\nu \frac{d u}{d \xi_{1}}\right) \frac{1}{r_{a}}-\chi(1+\nu) \theta\right] \frac{1}{1-\nu^{2}}
\end{aligned}
$$

where $\bar{\sigma}_{r}=\sigma_{r} / E$ and $\bar{\sigma}_{\phi}=\sigma_{\phi} / E$ are non-dimensional radial and tangential stresses, respectively. The near closed form solution for the stress field is obtained by combining Eq. (4.7) and Eq. (4.8)

$$
\begin{aligned}
& \bar{\sigma}_{r}=A \xi_{1}^{\eta_{1}-1}+B \xi_{1}^{\eta_{2}-1}-\chi \sum_{i=0}^{m} \frac{i L_{i}}{n(1+i+\nu)+i(i+2)} \xi_{1}^{i} \\
& \bar{\sigma}_{\phi}=-\eta_{2} A \xi_{1}^{\eta_{1}-1}-\eta_{1} B \xi_{1}^{\eta_{2}-1}-\chi \sum_{i=0}^{m} \frac{i(n+i+1) L_{i}}{n(1+i+\nu)+i(i+2)} \xi_{1}^{i}
\end{aligned}
$$

where

$$
A=\frac{D_{1}\left(\eta_{1}+\nu\right)}{r_{a}\left(1-\nu^{2}\right)} \quad B=\frac{D_{2}\left(\eta_{2}+\nu\right)}{r_{a}\left(1-\nu^{2}\right)}
$$

are constants estimated from the boundary conditions $\bar{\sigma}_{r}=0$ at $\xi_{1}=1$ and $R$.

\section{Results and discussion}

A near closed form solution for thermal stresses in the isotropic annular fin with a hyperbolic profile is derived. ADM is employed to obtain the non-dimensional temperature field represented in a polynomial form. The integral constants $C$ are evaluated using the minimum decomposition error $J=\theta_{i+1}-\theta_{i},\left(J \leqslant 10^{-4}\right)$ approach. Figure 2 represents the values of the integral constant $C$ for different values of the variable thermal conductivity parameter. The accepted $C$ values are the $x$-values corresponding to the minimum decomposition error in each case. These constants directly influence the variation of local temperature distribution. The determined values of $C$ for $\psi=0.2$ and $\beta=0.3,0$ and -0.3 are $-0.1737,-0.2167$ and -0.2758 , respectively.

With the best of literature search, this work is the first attempt to estimate thermal stresses in an annular fin with a hyperbolic profile along with a variable thermal conductivity. Due to this limitation, stress fields for the hyperbolic fin profile obtained from the present formulation could not be compared. However, the present formulation for stress fields can be used to obtain results for an annular fin of uniform thickness by setting $n=0$ in Eq. (4.9). These stress field results are compared with those by Chiu and Chen (2002) shown in Fig. 3. The results in Fig. 3 take into account the same parameters, $\kappa= \pm 0.00018, \xi_{1}=1$ to 3 , $h=50 \mathrm{~W} /\left(\mathrm{m}^{2} \mathrm{~K}\right), k_{\infty}=186 \mathrm{~W} /(\mathrm{mK})$ and $t\left(=h_{a}\right)=0.004 \mathrm{~m}$ and boundary conditions for clarity of the comparison. Both temperature and stress fields in uniform thickness obtained from Eqs. (4.5) and (4.9) visibly reveal close agreement.

Figure 4 illustrates the effect of various thermo-mechanical parameters on the non-dimensional temperature and stress field. Unless mentioned otherwise, the numerical values 


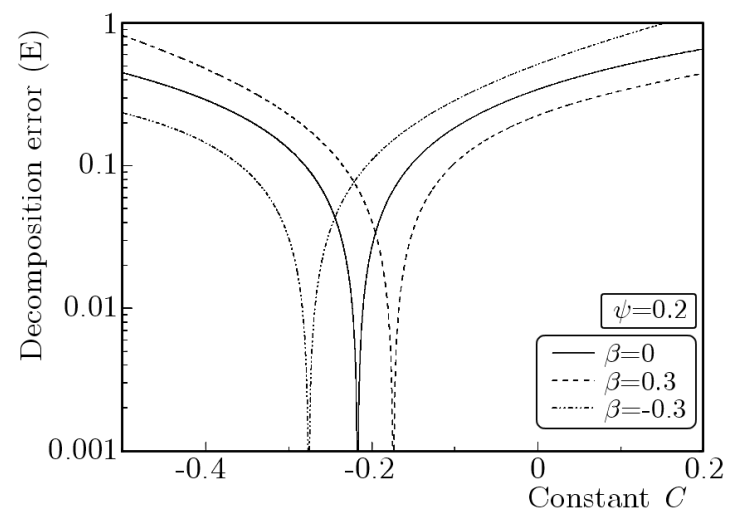

Fig. 2. Estimation of the integral constant using the minimum decomposition error approach
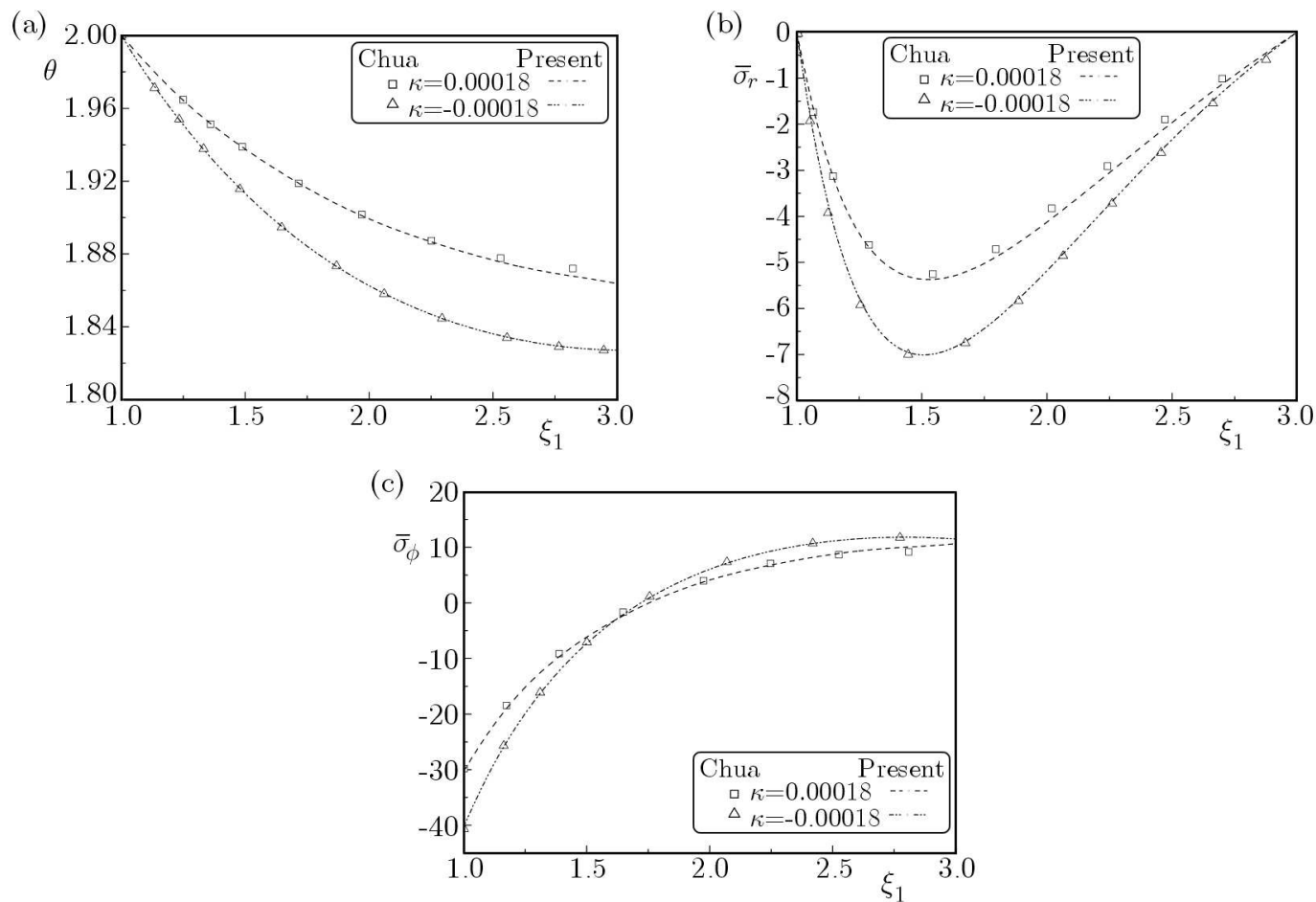

Fig. 3. Comparison of the results for (a) temperature distribution, (b) radial stress distribution and (c) tangential stress distribution for an annular fin with uniform thickness. The results heve been estimated from the present closed form solution for the fin with variable thickness by setting $n=0$

of the non-dimensional parameters are taken to be $\beta=0, \psi=0.2, \chi=1$ and $\nu=0.3$ for all the cases. In Fig. 4a, it can be seen that the steeper temperature gradient is associated with a lower variable thermal conductivity parameter $\beta$. As a result, the mean temperature difference between the base and fin tip is decreased with an increase in $\beta$. On the other hand, higher thermogeometric parameter $\psi$ induces higher temperature gradient. Lower thermo-geometric parameter indicates the fin as thermally thin with less thermal resistivity. Thus, the heat conduction inside the body is much faster than the heat convection away from the surface. Furthermore, it can be observed that the coefficient of thermal expansion $\chi$ and Poisson's ratio $\nu$ do not affect the temperature field. Nevertheless, the variation of stresses is influenced by all thermo-mechanical parameters. The stress field in Eq. (4.9) can be seen to vary linearly with the coefficient of thermal expansion. Consequently, the stress field can be changed with modification in the coefficient of thermal expansion by $n$-times. Thus, the maximum of non-dimensional radial and tangential 
stress magnitudes: -0.0558 and -0.19523 increase to -0.1126 and -0.39046 , respectively, by a change in the coefficient of thermal expansion from 1 to 2 (negative value indicates compressive stress) shown in Fig. 4b and 4c. The parameters $\beta$ and $\psi$ significantly influence the variation of stress fields. The stress magnitude increases with an increment of $\psi$, and decreases with an increase in $\beta$. Interestingly, the stress field is very marginally affected by Poisson's ratio $\nu$ (Fig. 4). This result is reasonable for axisymmetric plane stress assumptions in the analysis of the annular fin with a hyperbolic profile.
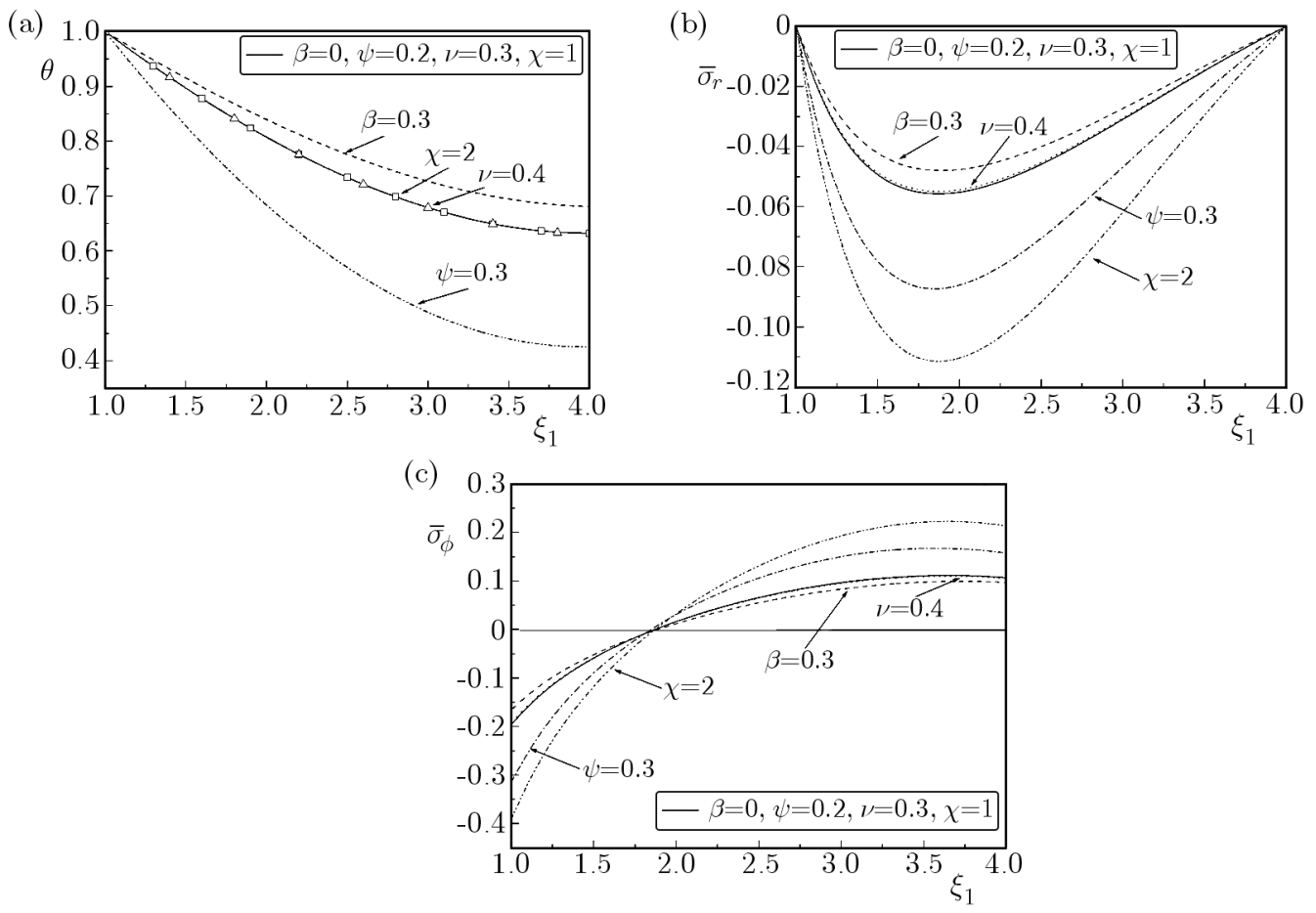

Fig. 4. Effect of various thermo-mechanical properties on (a) temperature distribution, (b) radial stress field, and (c) tangential stress field. Unless mentioned otherwise, $\beta=0, \psi=0.2, \chi=1$ and $\nu=0.3$

A comparison of the stress field between a fin of uniform thickness and that of a hyperbolic profile keeping the same material volume condition are depicted in Fig. 5a and 5b. Except for the profile geometry, all other parameters, i.e. $V=4.0212 \cdot 10^{-5} \mathrm{~m}^{3}, \kappa=0, h=50 \mathrm{~W} /\left(\mathrm{m}^{2} \mathrm{~K}\right)$, $k_{\infty}=186 \mathrm{~W} /(\mathrm{mK})$ (Chiu and Chen, 2002) are maintained the same in both profiles. Herein, the maximum value of $\bar{\sigma}_{r}$ and $\bar{\sigma}_{\phi}$ (compressive) are found to be less in the hyperbolic profile than in the uniform thickness condition. The $\bar{\sigma}_{r}$ variation with the length parameter indicates a better symmetric distribution over dimensionless radius in the hyperbolic profile than that of the uniform thickness profile.

Furthermore, the $\bar{\sigma}_{\phi}$ (compressive) variation in the hyperbolic profile is significantly lower compared to the uniform thickness case near to the base of the fin. A marginally higher $\bar{\sigma}_{\phi}$ (tensile) variation is observed in the hyperbolic profile near the fin tip. These results reveal that the fin with the hyperbolic profile is much safer from the material failure view point than that of the uniform thickness profile due to the lower stress level. Conversely, for the same stress field, the fin with the hyperbolic profile is much more compact and requires less material.

Figure 6 shows the surface plot of the temperature and stress field along the radial direction for variable $\beta$ and $\psi$. The non-dimensional temperature surfaces (Fig. 6a) reveal that the temperature difference $\left(\Delta \theta=\theta_{r_{a}}-\theta_{\infty}\right)$ from the base to tip gradually increases with a decrease in variable thermal conductivity parameters. On the other hand, the reverse nature is observed with the variation of the thermo-geometric parameter. The results obtained suggest that for a 
(a)

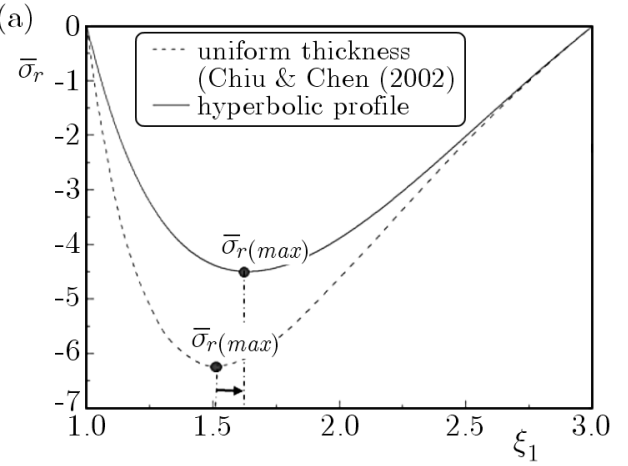

(b)

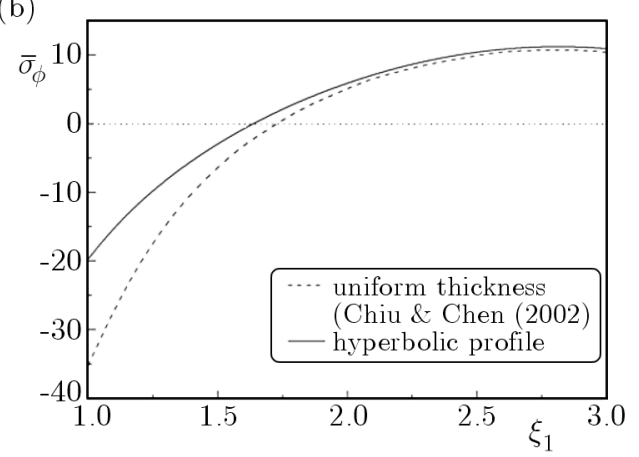

Fig. 5. Comparison of (a) radial stress field and (b) tangential stress field between the uniform and hyperbolic annular fin. The volume and other properties are the same in both cases

(i)

(a)

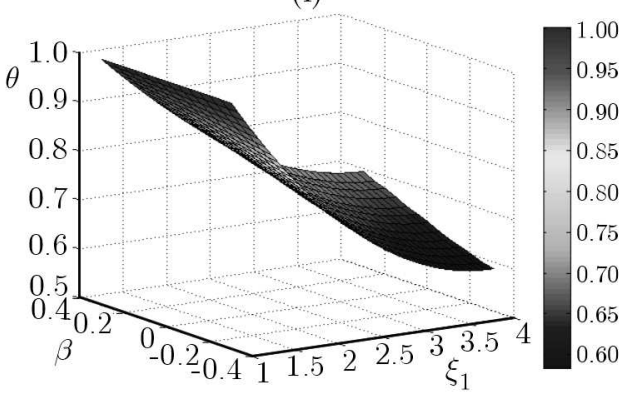

(b)

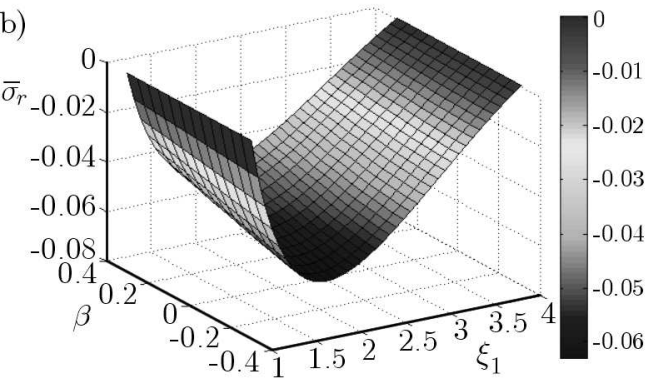

(c)

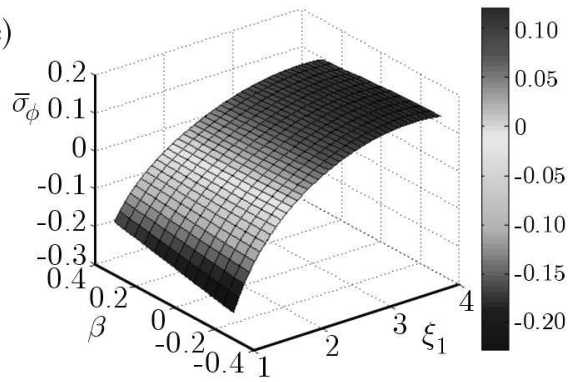

(ii)
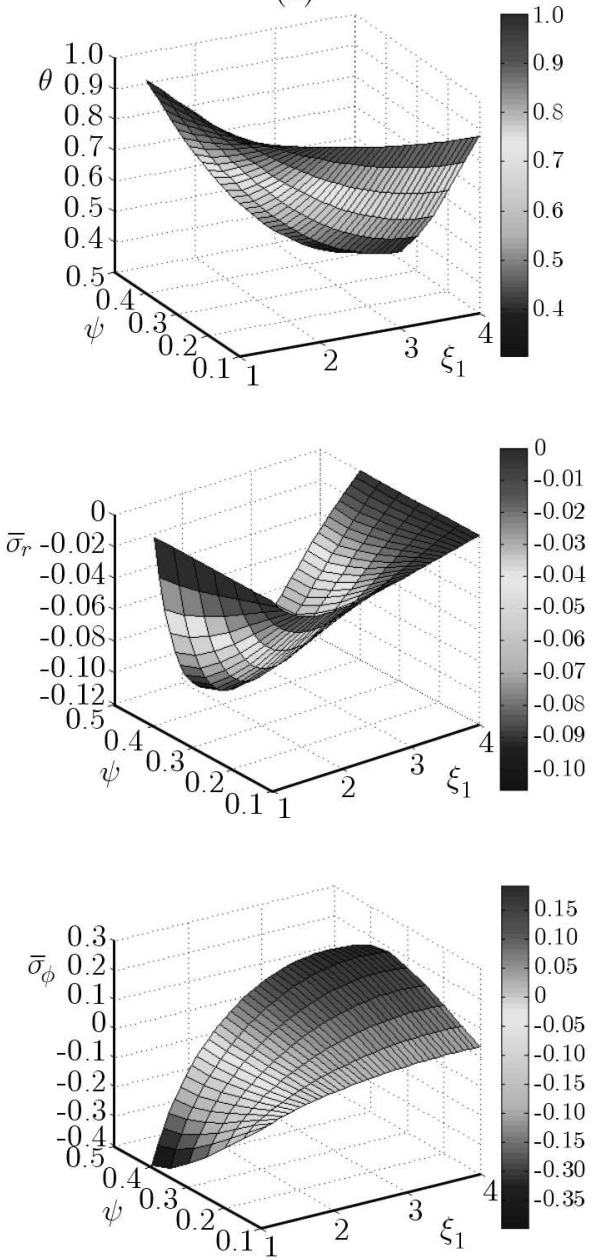

Fig. 6. Surface domain for (a) temperature field, (b) radial stress field, and (c) tangential stress field along the radial direction $\xi_{1}$ with the variation of thermal parameters (i) $\beta$ and (ii) $\psi$

specific surface geometry the heat transfer can be enhanced either by decreasing the thermogeometric parameter or by selecting materials with higher thermal conductivity. In addition to the heat transfer enhancement, the investigation of material failure is also an important aspect for the designer of the fin. The surface plots for $\bar{\sigma}_{r}$ and $\bar{\sigma}_{\phi}$ distribution with the variation of $\beta$ and $\psi$ are depicted in Fig. 6b and 6c, respectively. From the surface representation of stress distribution, it is apparent that the maximum $\bar{\sigma}_{r}$ (compressive) and $\bar{\sigma}_{\phi}$ (compressive or tensile) 
are achievable either with a lower value of $\beta$ or by the use of a higher thermo-geometric parameter $\psi$. The lower value of $\beta$ induces high thermal resistance. As a consequence, poor heat transfer through the fin material causes a higher value of the local temperature difference between two neighbouring material points along the radial direction. Thus, a very low heat transfer can lead to the excessively induced thermal stress that reduces the fin life. Therefore, a study of the maximum limit of heat dissipation ability and the maximum limit of thermal stresses induced is necessary for better design.

\section{Conclusions}

A method of analysis for the determination of thermal stresses in an annular fin with a hyperbolic profile using variable thermal conductivity is presented. The ADM coupled with thermo-elasticity approach is chosen to derive an approximate analytical solution for thermal stresses. In order to validate the present analytical solution, the results are compared with the results available in literature and found to be in very good agreement. The effects of various non-dimensional parameters on the temperature field and stress fields are also investigated. Based on the present study, the remarkable outcomes are:

i. ADM is useful and efficient to obtain a general closed form solution for the stress field in fin of a hyperbolic profile as well as of a uniform thickness with variable thermal conductivity.

ii. The temperature field is influenced by the parameters $\beta$ and $\psi$ only, while, the stress fields are governed by all non-dimensional parameters $\beta, \psi, \chi$ and $\nu$. Notably, the effect of Poisson's ratio $\nu$ on the stress field is observable only in hyperbolic fin profile. And this effect is very insignificant compared to the contribution of other parameters.

iii. The variation of radial stress with the length parameter exhibits better symmetric distribution in the hyperbolic profile then that of the uniform thickness.

iv. The surface plot for the temperature field and stress field with different values of $\beta$ and $\psi$ discloses the nature of thermo-parametric dependence on the temperature and stress field.

v. Improvement in heat transfer can be attained either by increasing the thermal conductivity parameter or setting a lower thermo-geometric parameter. The lower value of local temperature difference may induce higher thermal stresses in the part. So, an appropriate combination of the heat dissipation limit and induced stress field can only be achieved with an efficient design of the fin profile. The hyperbolic section of the fin appears to be a better choice in all respects.

vi. The present method of analysis is expected to help understanding of the heat transfer phenomena and thermal stress development in the hyperbolic fin.

\section{Appendix}

The $K_{i}$ estimation (14 terms considered in this study) are given below

$$
\begin{aligned}
K_{0} & =1 \quad K_{1}=C \\
K_{2} & =\frac{1}{2}\left(-C^{2} \beta+\psi^{2}+C^{2} \beta^{2}-\psi^{2} \beta-C^{2} \beta^{3}+\psi^{2} \beta^{2}+C^{2} \beta^{4}-\psi^{2} \beta^{3}\right) \\
K_{3} & =\frac{1}{6}\left(C \psi^{2}+\psi^{2}+3 C^{3} \beta^{2}-4 C \beta \psi^{2}-\beta \psi^{2}-6 C^{3} \beta^{3}+\beta^{2} \psi^{2}+7 C \beta^{2} \psi^{2}+7 C^{3} \beta^{4}\right. \\
& \left.-8 C \psi^{2} \beta^{3}-\psi^{2} \beta^{3}\right)
\end{aligned}
$$




$$
\begin{aligned}
K_{4} & =\frac{1}{24}\left(2 C \psi^{2}-5 C^{2} \beta \psi^{2}-6 C \beta \psi^{2}+\psi^{4}-15 C^{4} \beta^{3}+28 C^{2} \beta^{2} \psi^{2}+10 C \beta^{2} \psi^{2}-5 \beta \psi^{4}\right. \\
& \left.+32 C^{4} \beta^{4}+4 C^{3} \beta^{4}+C^{2} \beta^{3}-53 C^{2} \beta^{3} \psi^{2}-16 C \beta^{3} \psi^{2}-\beta^{2} \psi^{2}+7 \beta^{2} \psi^{4}\right) \\
K_{5} & =\frac{1}{120}\left(-13 C^{2} \beta \psi^{2}+C \psi^{4}+4 \psi^{4}+38 C^{3} \beta^{2} \psi^{2}+56 C^{2} \beta^{2} \psi^{2}-20 C \beta \psi^{4}-18 \beta \psi^{4}\right. \\
& \left.+78 C^{5} \beta^{4}+12 C^{4} \beta^{4}-205 C^{2} \beta^{3} \psi^{2}-120 C^{2} \beta^{3} \psi^{2}-2 C \beta^{3} \psi^{2}+96 C \beta^{2} \psi^{4}+50 \psi^{4}\right) \\
K_{6} & =\frac{1}{720}\left(6 C \psi^{4}+4 \psi^{4}+120 C^{3} \beta^{2} \psi^{2}-21 C^{2} \beta \psi^{4}-86 C \beta \psi^{4}-18 \beta \psi^{4}+\psi^{6}-116 C^{4} \beta^{3}\right. \\
& +30 C^{5} \beta^{4}-272 C^{3} \beta^{3}-91 C^{4} \beta^{3} \psi^{2}-156 C^{3} \beta^{3} \psi^{2}-20 C^{2} \beta^{3} \psi^{2}+193 C \beta^{2} \psi^{2} \\
& \left.-48 C^{3} \beta^{2} \psi^{2}+124 C^{2} \beta^{2} \psi^{4}+172 C \beta^{2} \psi^{4}+16 \beta^{2} \psi^{4}+22 \beta^{2} \psi^{2}-8 \beta \psi^{4}-12 \beta \psi^{6}\right) \\
K_{7} & =\frac{1}{5040}\left(10 C \psi^{4}-150 C^{2} \beta \psi^{4}-128 C \beta \psi^{4}+C \psi^{6}+9 \psi^{6}-1234 C^{4} \beta^{3} \psi^{2}+329 C^{3} \beta^{2} \psi^{4}\right. \\
& \left.+1848 C^{2} \beta^{2} \psi^{4}+584 C \beta^{2} \psi^{4}-82 C \beta \psi^{6}-112 C^{3} \beta^{3} \psi^{2}-151 \beta \psi^{6}\right) \\
K_{8} & =\frac{1}{40320}\left(-298 C^{2} \beta \psi^{4}+12 C \psi^{6}+28 \psi^{6}+5644 C^{3} \beta^{2} \psi^{4}+5822 C^{2} \beta^{2} \psi^{4}-243 C^{2} \beta \psi^{6}\right. \\
& -2020 C \beta \psi^{6}-1310 \beta \psi^{6}-240 C^{4} \beta^{3} \psi^{6}+42 C^{3} \beta^{2} \psi^{8}+172 C^{2} \beta^{2} \psi^{8}+36 C \beta^{2} \psi^{8} \\
& \left.-2 C \beta \psi^{10}+\psi^{8}\right) \\
K_{9} & =\frac{1}{362880}\left(52 C \psi^{6}+28 \psi^{6}+6792 C^{3} \beta^{2} \psi^{4}+256 C^{2} \beta^{2} \psi^{4}-1055 C^{2} \beta \psi^{6}-3022 C \beta \psi^{6}\right. \\
& \left.-686 \beta \psi^{6}+C \psi^{8}+16 \psi^{8}\right) \\
K_{10} & =\frac{1}{1814400}\left(40 C \psi^{6}+298 C^{3} \beta^{2} \psi^{4}-3529 C^{2} \beta \psi^{6}-2692 C \beta \psi^{6}+10 C \psi^{8}+50 \psi^{8}\right) \\
K_{11} & =\frac{1}{19958400}\left(-5053 C^{2} \beta \psi^{6}-28 C \beta \psi^{6}+80 C \psi^{8}+140 \psi^{8}\right) \\
K_{12} & =\frac{1}{11975040}\left(-4 C^{2} \beta \psi^{6}+15 C \psi^{8}+7 \psi^{8}\right) \\
K_{13} & =\frac{1}{7076160} C \psi^{8}
\end{aligned}
$$

\section{References}

1. Adomian G., 1988, Non-Linear Stochastic System Theory and Application to Physics, Kluwer Academic Publisher, Dordrecht

2. Aksoy I.G., 2013, Adomian decomposition method for heat conduction in an annular fin of hyperbolic profile with temperature dependent thermal conductivity, Journal of Thermal Science and Technology, 33, 1-8

3. Behnia M., Copeland D., Soodphadakee D., 1998, A comparison of heat sink geometries for laminar forced convection, Proceedings of the Sixth Intersociety Conference on Thermal and Thermomechanical Phenomena

4. Chiu C.H., Chen C.K., 2002, Application of the decomposition method to the thermal stresses in isotropic circular fins with temperature-dependent thermal conductivity, Acta Mechanica, 157, $147-158$

5. Kern Q.D., Kraus D.A., 1972, Extended Surface Heat Transfer, McGraw-Hill, New York, USA

6. Kraus A.D., Aziz A., Welty, J.R., 2001, Extended Surface Heat Transfer, John Wiley and Sons, New York

7. Mallick A., Ghosal S., Sarkar P.K., Ranjan R., 2015, Homotopy perturbation method for thermal stresses in an annular fin with variable thermal conductivity, Journal of Thermal Stresses, 38, 110-132 
8. Miansari M.O., Ganji D.D., Miansari M.E., 2008, Application of He's variational iteration method to nonlinear heat transfer equations, Physics Letters A, 372, 770-785

9. Mokheimer E.M.A., 2002, Performance of annular fins with different profiles subject to variable heat transfer coefficient, International Journal of Heat and Mass Transfer, 45, 3631-3642

10. Moradi A., 2011, Analytical solution for fin with temperature dependent heat transfer coefficient, International Journal of Applied Science and Engineering, 3, 1-12

11. Timoshenko S.P., Goodier J.N., 1970, Theory of Elasticity, McGraw-Hill, New York

12. YEH R.H., 1997, An analytical study of the optimum dimensions of rectangular fins and cylindrical pin fins, International Journal of Heat and Mass Transfer, 40, 3607-3615

Manuscript received May 29, 2015; accepted for print August 25, 2015 